\title{
An overview of concussion consensus statements since 2000
}

\author{
Robert C. Cantu, M.D. \\ Neurosurgery Service, Department of Surgery, and Sports Medicine Service, \\ Emerson Hospital, Concord, Massachusetts
}

\begin{abstract}
$\checkmark$ More refereed publications on sports-related concussion have appeared since 2000 than in all previous years combined. Three international consensus statements, documents from the National Athletic Trainers' Association (NATA) and the American College of Sports Medicine (ACSM), and entire issues of the Clinical Journal of Sport Medicine and the Journal of Athletic Training have been devoted to this subject. The object of this article is to critique the consensus statements and NATA and ACSM documents, pointing out areas of controversy.
\end{abstract}

\section{KEY WORDS • brain concussion • postconcussion syndrome • sports-related concussion - neuropsychological assessment • sports medicine}

I $\mathrm{T}$ has been estimated by PubMed Central that more refereed publications on sports-related concussion have appeared since the year 2000 than in all the previous years combined. Since 2000, three international concussion-in-sport consensus statements have been published: 1) the Vienna statement of $2001 ;{ }^{1}$ 2) the Prague statement of $2004 ; 5$ and, most recently, the St. Moritz conference of 2006. Extensive documents on concussion have also emerged from the NATA (the National Athletic Trainers' Association position statement, published in 2004) ${ }^{3}$ and the ACSM (a consensus statement on concussion and the team physician, which appeared in 2006). ${ }^{4}$ In addition, entire issues of the Clinical Journal of Sport Medicine (July 2001) and the Journal of Athletic Training (July-September 2001) have been devoted to the subject.

A consensus statement obviously is just that, but the consensus is often not unanimous. Whenever writing groups are assembled from diverse organizations as well as from diverse personal and professional backgrounds, organizational or personal bias may be present, no matter how much expertise is brought to the table. The purpose of this paper, written by a member of the writing groups of all three international concussion consensus statements as well as the NATA and ACSM publications, is to give an overview as well as a personal critique of each of these documents.

\section{Consensus Statements}

Summary and Agreement Statement of the First International Conference on Concussion in Sport, Vienna 2001

This document was published concurrently in early 2002 in the British Journal of Sports Medicine, the Clini-

Abbreviations used in this paper: $\mathrm{ACSM}=$ American College of Sports Medicine; FIFA = Fédération Internationale de Football Association; IOC = International Olympic Committee; NATA = National Athletic Trainers' Association. cal Journal of Sport Medicine, and Physician and Sports Medicine. ${ }^{1}$ (See Appendix 1 for lists of authors and affiliations.) The conference was held in November 2001 and was supported and organized by the International Ice Hockey Federation, the FIFA Medical Assessment and Research Center, and the IOC Medical Commission, with a stated objective of providing recommendations for the improvement of the safety and health of athletes who suffer concussive injuries in ice hockey, soccer, and other sports. Experts were invited to address specific issues involving epidemiology, basic and clinical science, grading systems, cognitive assessment, new research methods, protective equipment, management, prevention, and longterm outcome from concussive injury. At the conclusion of the conference, a small group of the experts was given the mandate to draft the document that was subsequently published in the three journals previously mentioned.

The Vienna conference was, in my opinion, very successful, and the resulting publication is the most comprehensive of the three international consensus statements on concussion in sport. I recommend it as a valuable resource for all who are interested in sports-related concussion.

This document provided a revised definition of concussion as "a complex pathophysiological process affecting the brain, induced by traumatic biomechanical forces." The statement further qualified the definition by noting five conclusions about the nature of concussive head injury. First, "concussion may be caused by a direct blow to the head, face, neck, or elsewhere on the body with an 'impulsive' force transmitted to the head." Second, a "concussion typically results in the rapid onset of short lived impairment of neurological function that resolves spontaneously." Third, "concussion may result in neuropathological changes but the acute clinical symptoms largely reflect a functional disturbance rather than structural injury." Fourth, "concussion results in a graded set of clinical syndromes that may or may not involve loss of consciousness. Resolution of the clinical and cognitive 
symptoms typically follows a sequential course." Fifth, "concussion is typically associated with grossly normal structural neuroimaging studies." All five of these conclusions are supported by current evidence.

No single concussion grading scale was endorsed by the conference-perhaps in part because experts who had produced grading scales of their own were at the writing table. In place of a single grading scale and in the absence of any scientifically validated return-to-play guidelines, the participants recommended the use of a clinical construct based on an assessment of recovery from injury and graded return to play. This construct includes the sideline evaluation of signs and symptoms of concussion as well as repeated assessments until all postconcussion symptoms resolve. It was recommended that a scale of postconcussion symptoms be used for both the initial sideline assessment and subsequent assessments. (See Appendices 2 and 3 for examples of a symptom checklist and a postconcussion symptom scale, respectively.) The conference participants concluded that sideline evaluation, including neurological assessment and mental status testing, is an essential component in evaluating sports-related concussion. I believe that these recommendations are sound and are not controversial.

Another consensus of this group was that "neuropsychological testing is one of the cornerstones of concussion evaluation and contributes significantly to both understanding of the injury and management of the individual." It was suggested that the computer-based programs Immediate Postconcussion Assessment and Cognitive Testing (ImPACT), CogSport, Automated Neuropsychological Assessment Metrics (ANAM), Sports Medicine Battery, and HeadMinder may have advantages over paper-and-pencil neuropsychological tests such as the McGill Abbreviated Concussion Evaluation (ACE) and the Standardized Assessment of Concussion (SAC). This is the first time that neuropsychological testing has been so strongly advocated in a concussion-in-sport consensus statement.

A new initiative that emerged from this group was the recommendation that return to play after concussion should follow a stepwise process. In this protocol, the injured athlete initially follows a regimen of complete rest until he or she is asymptomatic without activity. This initial stage is followed by one in which the athlete engages in light aerobic exercise, such as walking or stationary cycling. Having successfully completed that second stage, the athlete moves on to sport-specific training (for example, skating drills in hockey or running in soccer). The athlete then progresses from that third stage to a fourth one involving noncontact training drills, then to a fifth one in which fullcontact training drills are allowed before receiving medical clearance to return to game play. It was recommended that this stepwise progression be followed and that the athlete proceed to the next level if asymptomatic at the current level. If any symptoms occur after ascending to a given level, the athlete should drop back down to the level at which he or she had been asymptomatic.

Although I believe that this is a prudent protocol for cases in which athletes have been away from play for weeks or a month or more after a concussion, I do not think that this protocol is necessary for athletes who are allowed to return to play within a week.

Another observation to emerge from the Vienna confer- ence was that because the brain is not an organ that can be conditioned to withstand concussive injury, there are relatively few means by which such injury can be minimized in sports. Thus, perhaps the most important ways to reduce or prevent concussions are rule changes and rule enforcement. The authors acknowledged that the science of studying concussion is at an early stage and that, as a result, decisions regarding concussion management and return to play lie largely in the realm of clinical judgment and must be made on an individual basis.

The group concluded that whenever a player shows any symptoms or signs of concussion, he or she should not be allowed to return to play in the current game or practice, should not be left alone, should undergo regular monitoring for deterioration of condition, should undergo medical evaluation after the injury, and that return to play should follow a medically supervised, stepwise process. It was emphasized that the player should never return to play while symptomatic and that the adage "when in doubt, sit them out" should be followed. I believe these conclusions are sound, especially with regard to return-to-play issues.

National Athletic Trainers' Association Position Statement: Management of Sport-Related Concussion

In my opinion, this position statement, which was published in 2004 in the Journal of Athletic Training, ${ }^{3}$ is the most comprehensive document published to date on sportsrelated concussion. The writing team was chaired by Kevin Guskiewicz, Chair of the Department of Sport and Exercise Science at the University of North Carolina, and included seven other recognized experts in the field of concussion. The writing process took more than a year and resulted in a document that comprises 36 specific recommendations and contains sections on defining and recognizing concussion; evaluating and making return-to-play decisions; and assessment tools, including instruments for postconcussion symptom assessment, cognitive screening, postural stability assessment, and neuropsychological testing (with subsections devoted to computerized neuropsychological tests and neuropsychological testing methods). It also contains sections on when to refer an athlete to a physician after a concussion and when to disqualify an athlete, as well as sections on special considerations for the young athlete, home care, and equipment issues.

As a reference source on the entire subject of sport concussion as well as for its comprehensive bibliography source on sport concussion, I strongly endorse this document.

Summary and Agreement Statement of the 2nd International Conference on Concussion in Sport, Prague 2004

The same organizational bodies that convened the First International Conference on Concussion in Sport in Vienna in 2001, namely the International Ice Hockey Federation, FIFA, and the IOC, convened a second conference on concussion in sport in Prague in November 2004. At the conclusion of the Prague conference, a small writing group composed of the same individuals who were involved with the Vienna document of 2001 was given the task of drafting a document describing the agreement positions reached by those in attendance at the meeting on 
a variety of topics related to sport concussion. These topics included epidemiology, basic and clinical science, injury grading systems, cognitive assessment, new research methods, protective equipment, management, prevention, and long-term outcomes. Like the first document, this second document was published concurrently in the British Journal of Sports Medicine, the Clinical Journal of Sport Medicine, and Physician and Sports Medicine. ${ }^{5}$

This second international conference on concussion in sport was considerably more widely attended than the first and had a much greater representation from new groups, such as trauma surgeons and sports psychologists. The summary and agreement statement included a new pocket-sized, sideline-assessment summary card for use by clinicians. No significant breakthroughs in scientifically validated information on concussion had occurred between the two conferences; therefore, the second document might best be viewed as a modest updating of the first. One of the most meritorious recommendations from that statement was the one that concussion severity should only be determined after the following criteria have been met: 1) all signs and symptoms of concussion have cleared; 2) the results of neurological examination have returned to normal; and 3) the results of any neuropsychological tests or other cognitive function tests that might have been performed have returned to baseline or above.

Noting that brief loss of consciousness does not necessarily correlate with concussion severity; despite its association with early neuropsychological deficits, the participants recommended that loss of consciousness not be relied on as a measure of concussion severity. Another recommendation was that pediatric cases could be managed using guidelines similar to those used in caring for adult patients.

The authors also elaborated on the concept of "cognitive risks." They suggested that scholastic activities and activities of daily living be modified while an athlete is still symptomatic following a concussion, because vigorous pursuits might intensify or prolong postconcussion symptoms.

Perhaps the most contentious recommendation was the suggestion that concussions could be divided into the following two categories. 1) A case of simple concussion was defined as one in which neurological symptoms resolved within 7 to 10 days. 2) A case of complex concussion was defined as one in which symptoms persisted longer than 10 days or the patient lost consciousness for longer than 1 minute, had a convulsive concussion, or had repeated concussions involving diminishing force. The writing group's decision to use these two categories of concussion was not unanimous, because some membersincluding me-were certain that they would not refer to a concussion with symptoms lasting 10 days as a simple concussion. The word "simple" may, in fact, not be a good choice for describing any concussion.

Nonetheless, the group members agreed that the number, duration, and severity of total postconcussion symptoms were most important in determining concussion severity and that the combination of symptoms was more important than the single symptom of amnesia.

This document also described the motor phenomena, such as tonic posturing and convulsive movement, seen with convulsive concussion and emphasized that although the presentation is dramatic, the outcome is usually benign and this form of concussion requires no specific treatment beyond the usual concussion management.

Another less-than-unanimous conclusion was that neuropsychological assessment following concussion is definitely of value, but should not be performed until all signs and symptoms have resolved. A unanimous conclusion regarding neuropsychological testing was that it is one piece of the concussion management puzzle but should never be a sole criterion to determine when an athlete should be allowed to return to play.

The statements regarding concussion management and rehabilitation were largely the same as those found in the Vienna document.

\section{Concussion (Mild Traumatic Brain Injury) and the Team} Physician: A Consensus Statement

In 2006, the ACSM published a sports-related concussion consensus statement (Concussion [Mild Traumatic Brain Injury] and the Team Physician: A Consensus Statement) in Medicine Science and Sports and Exercise. ${ }^{4}$ Created as a reference tool for team physicians, this statement represented the collaborative effort of six major professional associations, including the American Academy of Family Physicians, the American Academy of Orthopedic Surgeons, the ACSM, the American Medical Society for Sports Medicine, the American Orthopedic Society for Sports Medicine, and the American Osteopathic Academy for Sports Medicine. The team physician consensus statement was endorsed by a number of additional organizations, including the American Osteopathic Association, the NATA, the North American Spine Society, the National Collegiate Athletic Association, the National Youth Sports Safety Foundation, the American Academy of Podiatric Sports Medicine, and the American Kinesiotherapy Association. The expert writing panel was chaired by Stanley A. Herring. I believe this is an extremely useful document. The major goal of the writing group was to provide an overview of selected medical issues that are of keen importance to team physicians responsible for athletes with concussion. The areas covered include concussion epidemiology, pathophysiology, game-day evaluation and treatment, post-game-day evaluation and treatment, diagnostic imaging, management principles, return-to-play decisions, complications of concussion, and prevention. Each section of the document begins with the panel's consensus on what is essential and continues with what is desirable for the team physician to know and understand. This is the feature of this document that I personally find most useful. Categorized as essential were methods of recognizing and evaluating athletes with concussion, the necessity for individualized management and treatment of athletes with concussion, the need for return-to-play decisions to be based on clinical judgment, the importance of developing a game-day medical plan specific to concussion injury, and the necessity of documentation. The paucity of well-designed studies of concussion and its natural history was also noted. It was concluded that it would be desirable for the team physician to have the ability to coordinate a systematic approach for the treatment of athletes with concussion, to identify risk factors and implement appropriate treatment, to under- 
stand the potential sequelae of concussive injuries, and to understand preventive strategies.

For a team physician, another extremely useful aspect of this document was that it was broken down in terms of game-day treatment (treatment on the field as well as treatment on the sideline) and post-game-day treatment, in terms of concussion evaluation, management, and return-to-play criteria. It was considered essential that before any athlete be allowed to return to play, he or she should be asymptomatic at rest and exertion and must remain asymptomatic with exertion. Other factors that were considered essential in return-to-play decisions, especially post-game-day decisions, were the severity of the current injury as documented primarily by the number and duration of postconcussion symptoms; the number, severity, and proximity of previous concussions; whether a severe injury had occurred in response to what appeared to be a minor blow; the age of the athlete (with heightened concern for trauma to the immature brain); the sport involved; and whether the athlete had any learning disabilities. In addition to persistent postconcussion symptoms, the following were considered contraindications to return to sport: abnormal results on neurological examination, signs or symptoms of concussion that manifest on exertion, and significant abnormalities on cognitive testing or imaging studies. This document encouraged team physicians to coordinate a group of individuals to implement progressive aerobic and resistive exercise challenge tests that the injured athlete would have to pass prior to full return to play and to recognize that challenging cognitive effort may exacerbate symptoms of concussion and retard recovery. Furthermore, it was recommended that the team physician discuss the status of injured athletes with parents, caregivers, teachers, certified athletic trainers, and the coaching staff within disclosure regulations.

Again, I found this to be a very useful document, especially for physicians on the sideline responsible for athletes who may have a cerebral concussion. This statement was similar to the Vienna and Prague documents in that it emphasized that concussion severity should be determined by the duration and number of postconcussion symptoms, not by whether there was brief loss of consciousness or even whether amnesia alone was one of the symptoms. This means that concussion severity should not be determined in most cases on the day of concussion, but rather only after all symptoms have resolved.

\section{International Conference on Concussion in Sport, St. Moritz 2006}

The final document to be briefly mentioned in this paper will be based on a conference that was held this past spring in St. Moritz, Switzerland. Because it is currently being written, the comments included here must be limited.

The document will focus at least in part on three areas in which there is some concern regarding the Prague statement. The first involves neuropsychological testing and its use, including the question of when it should be used in the management and assessment of concussion. The second involves the concept of simple versus complex concussion; and the third involves the question of whether the same concussion protocols should be followed in the management of cases involving young athletes as are followed in cases involving adult athletes. Although many other areas will be covered in the forthcoming document, these are three in which there may be significant variance from what was stated in the Prague document.

\section{Conclusions}

This subject is perhaps best summarized by the final comment in the 2004 Prague summary, which states, "This protocol represents a work in progress, and, as with all other recommendations or proposals, it must be updated as new information is added to the current state of the literature and understanding of this injury."

\section{Appendix 1}

Summary and Agreement Statement of the First International Conference on Concussion in Sport, Vienna 2001 and

Summary and Agreement Statement of the 2nd International Conference on Concussion in Sport, Prague 2004

Writing group members:

M. Aubry, Chief Medical Officer, International Ice Hockey Federation (IIHF)

R. Cantu, Chief, Neurosurgery Service and Director, Sports Medicine Service, Emerson Hospital, Concord, Massachusetts; Medical Director, National Center for Catastrophic Sports Injury Research, Chapel Hill, North Carolina

J. Dvorak, Chairman, FIFA Medical Research and Assessment Center (F-MARC); Wilhelm Neurologist and Director, Schulthess

Clinic, Zurich, Switzerland

T. Graf-Baumann, F-MARC, Tenningen, Germany

K. Johnston, Chair, Concussion in Sport Group, FIFA, IIHF, IOC; Neurosurgeon and Director of Neurotrauma, McGill University Health Centre, McGill University and McGill Sport Medicine Centre, Montreal, Canada

J. Kelly, Associate Professor of Clinical Neurology, Northwestern University Medical School, Chicago Neurological Institute, Chicago, Illinois

M. Lovell, Director, Sports Medicine Concussion Program, University of Pittsburgh; Co-Director, National Hockey League Neuropsychology Program, Pittsburgh, Pennsylvania

P. McCrory, The Brain Research Institute and Center for Sports Medicine Research and Education, University of Melbourne, Australia

W. Meeuwisse, University of Calgary Sport Medicine Centre, Sport Injury Consultant, National Hockey League, Calgary, Alberta, Canada

P. Schamasch, Director, IOC Medical Commission, Lausanne, Switzerland

National Athletic Trainers' Association Position Statement:

Management of Sport-Related Concussion

Writing committee:

Kevin M. Guskiewicz, Ph.D., Chair, Department of Sport and Exercise Science, University of North Carolina, Chapel Hill, North Carolina

Scott L. Bruce, M.S., California University of Pennsylvania, California, Pennsylvania

Robert C. Cantu, M.D., Emerson Hospital, Concord, Massachusetts

Michael S. Ferrara, Ph.D., University of Georgia, Athens, Georgia

James P. Kelly, M.D., University of Colorado, Denver, Colorado Michael McCrea, Ph.D., Waukesha Memorial Hospital, Waukesha, Wisconsin

Margot Putukian, M.D., Princeton University, Princeton, New Jersey

Tamara C. Valovich McLeod, Ph.D., Arizona School of Health Sciences, Mesa, Arizona 


\section{Concussion consensus statements}

\section{Concussion (Mild Traumatic Brain Injury) and the Team Physician: A Consensus Statement}

Expert panel:

Stanley A. Herring, M.D., Chair, Seattle, Washington

John A. Bergfeld, M.D., Cleveland, Ohio

Arthur Boland, M.D., Boston, Massachusetts

Lori A. Boyajian-O'Neill, D.O., Kansas City, Missouri

Robert C. Cantu, M.D., Concord, Massachusetts

Elliott Hershman, M.D., New York, New York

Peter Indelicato, M.D., Gainesville, Florida

Rebecca Jaffe, M.D., Wilmington, Delaware

W. Ben Kibler, M.D., Lexington, Kentucky

Douglas D. McKeag, M.D., Indianapolis, Indiana

Robert Pallay, M.D., Hillsborough, New Jersey

Margot Putukian, M.D., Princeton, New Jersey

Appendix 2

Postconcussion signs/symptoms checklist (symptoms at time of concussion)

bell rung
depression
dinged
dizziness
drowsiness
excessive sleep
fatigue
feel "in a fog"
feel "slowed down"
headache
irritability
loss of consciousness
memory problems
nausea
nervousness
numbness/tingling
poor Balance
poor Concentration
pinging in the ears
padness
pensitivity to Light
pensitivity to Noise
trouble Falling Asleep
vacant stare/glassy eyed
vomiting

Appendix 3

Cantu Evidence-Based Grading System for Concussion

\begin{tabular}{|c|c|}
\hline Grade 1 (mild) & No LOC $*$, PTA $\dagger<30 \mathrm{~min}, \ddagger \mathrm{PCSS}<24 \mathrm{~h}$ \\
\hline Grade 2 (moderate) & $\begin{array}{l}\text { LOC }<1 \text { min or PTA } \geq 30 \mathrm{~min}<24 \mathrm{~h} \text { or PCSS } \\
\geq 24 \mathrm{~h}<7 \mathrm{~d}\end{array}$ \\
\hline Grade 3 (severe) & $\mathrm{LOC} \geq 1 \mathrm{~min}$ or $\mathrm{PTA} \geq 24 \mathrm{~h}$ or $\mathrm{PCSS} \geq 7 \mathrm{~d}$ \\
\hline
\end{tabular}

(Reprinted with permission from Guskiewicz KM, Bruce SL, Cantu RC, Ferrara MS, Kelly JP, McCrea M, et al: National Athletic Trainers' Association position statement: management of sportrelated concussion. Athl Train 39:280-297, 2004.)

\section{References}

1. Aubry M, Cantu R, Dvorak J, Graf-Baumann T, Johnston K, Kelly J, et al: Summary and agreement statement of the first International Conference on Concussion in Sport, Vienna 2001. Br J Sports Med 36:6-10, 2002 (copublished in Clin J Sport Med 12:6-11, 2002; Phys Sportsmed 30:57-63, 2002)

2. Cantu RC: Concussion severity should not be determined until all post concussion symptoms have abated. Lancet Neurology 3:437-438, 2004

3. Guskiewicz KM, Bruce SL, Cantu RC, Ferrara MS, Kelly JP, McCrea M, et al: National Athletic Trainers' Association position statement: management of sport-related concussion. J Athl Training 39:280-297, 2004

4. Herring SA, Bergfeld JA, Boland A, Boyajian-O'Neill LA, Cantu RC, Hershman E, et al: Concussion (mild traumatic brain injury) and the team physician: a consensus statement. Med Sci Sports Exerc 38:395-398, 2006

5. McCrory P, Johnston K, Meeuwisse W, Aubry M, Cantu R, Dvorak J, et al: Summary and agreement statement of the 2nd International Conference on Concussion in Sport, Prague 2004 Br J Sports Med 39:196-204, 2005 (copublished in Clin J Sport Med 15:48-56, 2005; Phys Sportsmed 33:29-44, 2005)

Manuscript received July 6, 2006.

Accepted in final form September 11, 2006.

Address reprint requests to: Robert C. Cantu, M.D., Neurosurgery Service, Emerson Hospital, 131 Old Road to Nine Acre Corner, John Cuming Building, Suite 820, Concord, Massachusetts 01742. email: rcantu@emersonhosp.org. 
Author Names, et al. 